\title{
Quantitative Evaluation of Real-Time Shear-Wave Elastography under Deep Learning in Children with Chronic Kidney Disease
}

\author{
Jie Zhang $\mathbb{D}^{1},{ }^{1}$ Cuirong Duan $\mathbb{D}^{2},{ }^{2}$ Xingxing Duan $\mathbb{D}^{1},{ }^{1}$ Yuan Hu $\mathbb{D}^{1},{ }^{1}$ Jinqiao Liu $\mathbb{D}^{1},{ }^{1}$ \\ and Wenjuan Chen $\mathbb{D}^{1}$ \\ ${ }^{1}$ Department of Ultrasound, Hunan Children's Hospital, Changsha 410007, Hunan, China \\ ${ }^{2}$ Department of Renal Rheumatology, Hunan Children's Hospital, Changsha 410007, Hunan, China \\ Correspondence should be addressed to Wenjuan Chen; 2018060000022@jlxy.nju.edu.cn
}

Received 18 October 2021; Revised 18 December 2021; Accepted 22 December 2021; Published 6 January 2022

Academic Editor: M Pallikonda Rajasekaran

Copyright (c) 2022 Jie Zhang et al. This is an open access article distributed under the Creative Commons Attribution License, which permits unrestricted use, distribution, and reproduction in any medium, provided the original work is properly cited.

\begin{abstract}
Objective. This research was to study the application value of real-time shear wave elastography (SWE) quantitative evaluation based on deep learning (DL) in the diagnosis of chronic kidney disease (CKD) in children. Methods. 60 children with pathological diagnoses of CKD were selected as a CKD group. During the same period, 45 healthy children for physical examination were selected as the control group. The application value of real-time shear-wave elastography based on DL in the evaluation of CKD in children was explored by comparing the differences between the two groups. Results. It was found that the elastic modulus values of the middle and lower parenchyma of the left kidney and right kidney in the case group were $(22.02 \pm 10.98) \mathrm{kPa}$ and $(21.99 \pm 11.87) \mathrm{kPa}$, respectively, which were substantially higher compared with $(4.61 \pm 0.47) \mathrm{kPa}$ and $(4.50 \pm 0.59) \mathrm{kPa}$ in the control group. Young's modulus (YM) of the middle and lower parenchyma of the left kidney in patients with CKD stages 3 to 5 was $13.27 \pm 0.83,24.21 \pm 5.69$, and $31.67 \pm 3.82$, respectively, and that of the right kidney was $17.26 \pm 0.98,26.76 \pm 7.22$, and $32.37 \pm 4.27$, respectively, and the difference was significant $(P<0.05)$. In patients with moderate and severe $C K D$, the YM values of the middle and lower parenchyma of the left kidney were $17.27 \pm 0.83,27.93 \pm 6.49$, and those of the right kidney were $17.26 \pm 0.98,29.56 \pm 6.49$, respectively, and the difference was statistically significant $(P<0.05)$. The serum creatinine $(\mathrm{Scr})$ of the $\mathrm{CKD}$ group was substantially higher than that of the control group, and the estimated glomerular filtration rate (eGFR) level of the former was lower than that of the latter. However, there was no statistical difference between the YM values of the middle and lower parts of the left and right kidneys of the CKD group and the control group. Conclusion. The DL-based SWE is a new noninvasive, real-time, and quantitative detection method, which can effectively evaluate the stiffness of the kidney and help to better detect the progress of CKD as a clinical reference.
\end{abstract}

\section{Introduction}

Chronic kidney disease (CKD) refers to the pathological process of excessive damage and repair of the kidney due to various pathogenic factors, such as inflammation, damage, bacteria, and immunity, resulting in renal fibrosis, renal insufficiency, and even renal failure, and the course of disease often exceeds three months [1]. It is one of the common types of pediatric urologic diseases. According to its development, once CKD progresses to end-stage renal disease, kidney replacement must be used for treatment, which is labor-intensive and expensive. Both families and society bear a huge burden, which has a serious impact on the physical health of children [2]. Although there are many clinical detection techniques for CKD, which can detect the degree of kidney damage to a certain extent, they are mostly limited [3]. The diagnostic sensitivity of using biochemical indexes as diagnostic criteria is weak, requiring repeated measurement and avoiding various influencing factors, which takes a long time to detect kidney abnormalities, thus delaying the development of the disease. Percutaneous renal biopsy is the gold standard for clinical diagnosis of CKD, but its invasive nature may lead to all other complications. In severe cases, it can lead to renal insufficiency and an inability 
to dynamically monitor the CKD progression, reducing patient compliance. Imaging detection is one of the very important auxiliary diagnostic and therapeutic means. Although computed tomography (CT) and magnetic resonance imaging (MRI) can detect the degree of kidney injury to a certain extent, they are very limited. In the screening process, neither method will be the first choice, and it is difficult to be widely promoted in clinical practice $[4,5]$.

In recent years, physical examination and conventional ultrasound have been mostly used in the diagnosis of children with CKD. Due to their advantages of noninvasiveness, simple examination methods, low price, and strong sustainability, they are easily accepted by most children and their parents. Compared with inspection, the advantages are considerable [6]. However, due to the interference of the child's physiological conditions and the shooting environment, there are certain differences in the ultrasound images obtained. However, the ultrasound image segmentation methods currently used in clinical practice are deep learning (DL) methods based on convolutional neural networks (CNN) [7]. Image segmentation techniques are similar, and it is difficult to improve the accuracy and efficiency of segmentation. In recent years, the FCN (fully convolutional network) has applied a new image segmentation algorithm based on DL and harvested good results [8]. The shear wave elastography (SWE) technology based on DL is a new noninvasive detection method for renal fibrosis, which makes up for the shortcomings of traditional detection methods [9]. The SWE technology is adopted to screen for CKD, assess and stage the disease, and diagnose early changes in the structure and function of the kidneys in CKD. It can effectively avoid and delay the development of diseases and improve the quality of life of children.

Therefore, real-time SWE based on DL was applied to evaluate children's kidneys, and compared with traditional ultrasound, to explore its diagnostic effect in children's chronic kidney disease and provide data support for its clinical application.

\section{Research Materials and Methods}

2.1. Research Objects. In the CKD group, 60 children with pathological diagnosis of CKD, who were admitted to hospital from May 2018 to January 2020 were selected as the research objects, aged $7.2 \pm 1.23$ years old. For the control group, to reduce the influence of other factors, 45 children without CKD were selected from the hospital during the same time, aged $8.1 \pm 2.01$ years old. It was ensured that all data and biochemical parameters were complete for all children enrolled in the study and those biochemical parameters were collected for all subjects within one week before and after the SWE examination. Then, a database was established to compare the data within and between groups and process and analyze data.

The inclusion criteria of the CKD group were as follows: (1) patients found to have renal function impairment after admission to the hospital for ultrasound examination and were initially diagnosed as CKD; (2) can be collection complete data; and (3) the child about to undergo a needle biopsy. Exclusion criteria: (1) preliminary examination found hypertension, autoimmune diseases, diabetes, etc.; (2) ultrasonic elastic imaging was unqualified; (3) obese children who cannot carry out the above examination.

The inclusion criteria of the control group were normal blood routine, liver, and kidney function, electrocardiogram, and urine test, and no systemic diseases such as diabetes, heart failure, hypertension, and kidney disease.

The children and their families included in the study had signed informed consent, and the test process had been approved by the ethics committee of the hospital.

2.2. Data Collection. Detailed information of subjects was collected, including their height, weight, age, and gender, and their body mass index (BMI) was accurately recorded. The biochemical indexes of renal function were measured within one week, including their serum creatinine, proteinuria, and urea nitrogen. During the urine test, it was necessary to keep the subject quiet and exclude other influencing factors, such as strenuous exercise, fever, and infection.

\subsection{Research Methods}

2.3.1. Chronic Kidney Disease Staging Standard. CKD is kidney damage or glomerular filtration rate (estimate glomerular filtration rate, eGFR) $<60 \mathrm{~mL} / \mathrm{min} / 1.73 \mathrm{~m}^{2}$ for more than three months, regardless of the factor. The kidney damage in the CKD group was scored. From the damage of their glomeruli, blood vessels, and the renal interstitium, they were classified into the following groups. Group 1: mild $($ score $<9)$, group 2: moderate $(9 \leq$ score $<18)$, group 3: severe (score $\geq 18$ ) [10]. The scoring criteria are shown in Table 1.

According to eGFR, CKD is classified into the following five groups. (1) CKD stage I: the eGFR level is normal or slightly increased (eGFR $90 \mathrm{~mL} / \mathrm{min} / 1.73 \mathrm{~m}^{2}$ ). (2) CKD stage II: the level of eGFR decreases slightly (eGFR: $60-89 \mathrm{~mL} /$ $\min / 1.73 \mathrm{~m}^{2}$ ). (3) CKD stage III: the level of eGFR is moderately decreased (eGFR: $30-59 \mathrm{~mL} / \mathrm{min} / 1.73 \mathrm{~m}^{2}$ ). (4) CKD stage IV: the eGFR level drops severely (eGFR: $15-29 \mathrm{~mL} / \mathrm{min} / 1.73 \mathrm{~m}^{2}$ ). (5) $\mathrm{CKD}$ stage $\mathrm{V}$ : renal failure $\left(\mathrm{eGFR}<15 \mathrm{~mL} / \mathrm{min} / 1.73 \mathrm{~m}^{2}\right)$ [11]. In addition, patients with CKD stage I and CKD stage II can only be diagnosed when urine protein/creatinine $>30 \mathrm{mg} / \mathrm{g}$ and the disease course has been longer than three months [12].

\subsubsection{Criteria for Pathological Staging of Membranous} Nephropathy. Pathological staging criteria for membranous nephropathy. Stage I: the glomerular capillaries are not abnormal in the light microscope. Electron microscopy showed that there were scattered or regularly distributed small immune complex-like electron-dense deposits in the foot process gap between the glomerular basement membrane and epithelial cells. Stage II: according to an optical microscopy, glomerular capillaries are unevenly thickened, forming "spikes". Electron microscopy showed that there were electron-dense deposits in epithelial cells. Stage III: 
TABLE 1: Kidney damage scoring standard (NA= not applicable).

\begin{tabular}{|c|c|c|c|c|}
\hline & 1 & 2 & 3 & 4 \\
\hline Glomerular hyperplasia & $<25 \%$ & $25 \%-50 \%$ & $>50 \%-75 \%$ & $>75 \%$ \\
\hline Staged glomerular damage & $<10 \%$ & $10 \%-25 \%$ & $>25 \%-50 \%$ & $>50 \%$ \\
\hline Glomerulosclerosis & $<10 \%$ & $10 \%-25 \%$ & $>25 \%-50 \%$ & $>50 \%$ \\
\hline Renal interstitial inflammatory cell infiltration & $<25 \%$ & $25 \%-50 \%$ & $>50 \%$ & NA \\
\hline Renal interstitial fibrosis & $<25 \%$ & $25 \%-50 \%$ & $>50 \%$ & NA \\
\hline Renal tubule atrophy & $<25 \%$ & $25 \%-50 \%$ & $>50 \%$ & NA \\
\hline Thickening of blood vessel walls & $<10 \%$ & $10 \%-25 \%$ & $>25 \%$ & NA \\
\hline Arterial transparency changes & $<10 \%$ & $10 \%-25 \%$ & $>25 \%$ & NA \\
\hline
\end{tabular}

according to the optical microscope, the newly formed base film-like substance surrounds the deposits, forming a network or chain. Electron microscopy revealed that the electron-dense substances were deposited under the basement membrane or epithelial cells. Stage IV: according to the optical microscopy, the basement membrane thickened greatly. Electron microscopy showed that the deposit lost the electron density, and irregular electron translucent areas appeared in the irregularly thickened base film [13].

2.3.3. Inspection Methods. For routine ultrasound, subjects were required to take a supine position after urination, breathe in and hold their breath for a two-dimensional ultrasound examination. The coronal section of the renal hilum was observed, the thickness, length, shape, and parenchymal echo of the kidney were measured, and the data were recorded [14]. Shear wave elastic ultrasound based on DL was performed in the lateral decubitus position. After inhaling, the patient should hold his/her breath, the maximum coronal long axis section of the kidney was taken, and shear wave elastic imaging was started. The tester breathed again and held his breath. The image was frozen after the elastic image was filled with more than two-thirds of the color and evenly distributed. The quantitative analysis of tissue elasticity was completed by Q-box, the test area was a circular graph that could adjust the size according to the demand, and the renal parenchyma area was placed under the renal capsule with the same diameter. Then, the elastic value of the regional tissue was calculated by computer. Young's modulus (YM), which was expressed in $\mathrm{kPa}$, was used to measure the elasticity, the image was stored, and the data was recorded. In the calculation of YM for kidneys, errors caused by other factors should be reduced as far as possible. The left kidney was measured five times and the right kidney was the same, and then the average value was taken to compare the cortical YM values between them [15].

\subsection{SWE Technology Based on DL}

2.4.1. DL Model. The CNN convolutional layer is connected to the fully connected layer, the FCN convolutional layer is still connected to the convolutional layer, and the output is a feature map of the same size as the input. The FCN classifies the pixels of the image, thus solving the problem of image segmentation at the semantic level. Unlike the traditional CNN convolutional layers, the FCN can accept input images of any size. The deconvolution layer is used to up-sample the feature map of the last convolution layer to restore it to the same size as the input image so that a prediction can be generated for each pixel. The spatial information in the original input image is preserved, and finally, the parity is classified in the up-sampled feature map [16]. The network structure is shown in Figure 1. The input can be a color image of any size. The output is the same size as the input, and the depth is 20 types of targets + background $=21$. It is performed on the PASCAL data set, and PASCAL has a total of 20 categories.

2.4.2. Ultrasound Image Evaluation. In this study, three indicators of precision, Dice coefficient, and sensitivity were used to evaluate ultrasound images [17]. The consistency between the segmentation results and the gold standard (GT) of ordinary elastic ultrasonic segmentation was calculated. In Figure 2, the Qseg represents the divided tissue area, which is equal to TP + FP, and the Qcr represents the standard tissue area, which is equal to $\mathrm{TP}+\mathrm{FN}$. The TP represents the number of pixels correctly classified as positive in Qseg, and the TN represents the number of pixels correctly classified as negative in non-Qseg. The FP represents the number of pixels incorrectly classified as positive in Qseg, and the FN represents the number of pixels incorrectly classified as negative in non-Qseg. The Dice coefficient measures the degree of spatial coincidence between the divided tissue area and the standard tissue area, that is, the degree of spatial coincidence between Qseg and Qcr, and is defined as shown in formula (1). Precision measures the actual proportion of positive pixels in the segmented tissue area, that is, the proportion of TP to Qseg, which is defined as shown in formula (2). Sensitivity measures the proportion of correctly segmented standard tissue regions, that is, the proportion of TP to Qcr, and is defined as shown in formula (3). The evaluation values of the three indicators are all in [0, 1]. The larger the value, the higher the consistency between Qseg and Qcr, and better the segmentation result.

$$
\begin{aligned}
\text { Dice } & =\frac{2 \times(\Omega \operatorname{Seg} \cap \Omega G r)}{\Omega \operatorname{Seg}+\Omega G r} \\
& =\frac{2 \times \mathrm{TP}}{2 \times \mathrm{TP}+\mathrm{FP}+\mathrm{FN}}, \\
\text { Precision } & =\frac{\Omega \operatorname{Seg} \cap \Omega G r}{\Omega \operatorname{Seg}} \\
& =\frac{\mathrm{TP}}{\mathrm{TP}+\mathrm{FP}},
\end{aligned}
$$




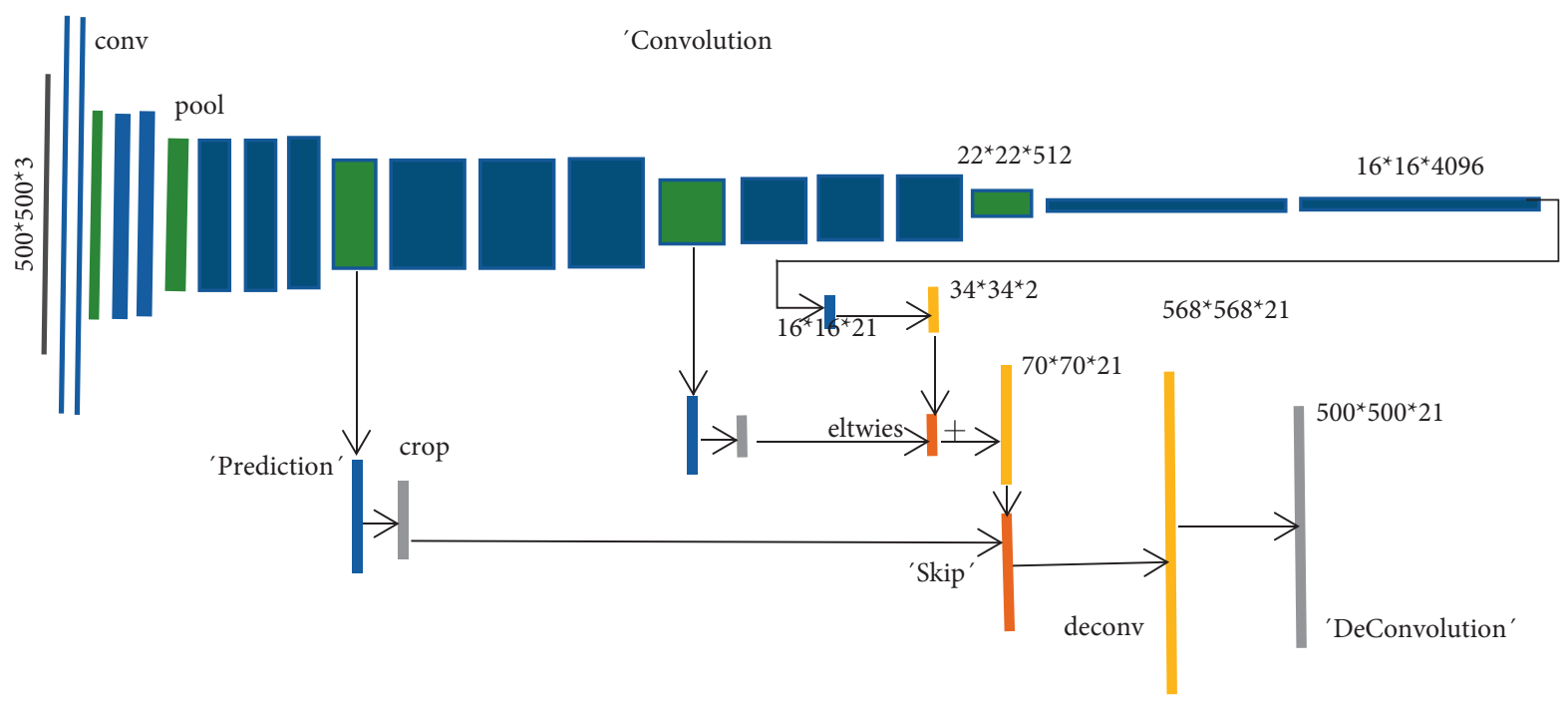

FIGURE 1: FCN network structure diagram.

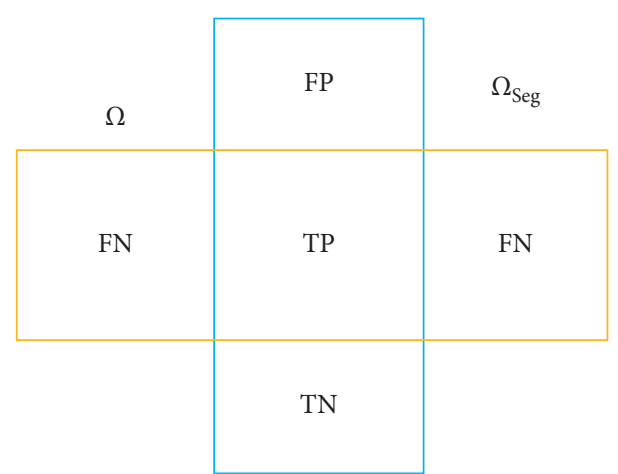

Figure 2: A schematic diagram of the two regions Qseg (blue box) and Qcr (orange box) obtained from the segmentation result and the gold standard (GT), respectively. TP (true), TN (true negative), FP (false positive), and FN (false negative number of pixels).

$$
\begin{aligned}
\text { Sensitivity } & =\frac{\Omega \operatorname{Seg} \cap \Omega G r}{\Omega G r} \\
& =\frac{\mathrm{TP}}{\mathrm{TP}+\mathrm{FN}} .
\end{aligned}
$$

2.5. Statistical Analysis. SPSS22.0 data processing software was used to classify and sort the collected data. The data that met the normal distribution were expressed by $(\overline{\mathrm{x}} \pm s)$. T-test was used to compare the results between the pairs. Wilcoxon's rank-sum test was used to analyze the YM value of the lower middle kidney of CKD patients at different stages and the lower middle kidney parenchyma of left and right kidneys compared with the control group. Pearson correlation was used to analyze the correlation between the YM value and laboratory indicators. $P<0.05$ was regarded as the criterion of statistical significance.

\section{Results}

3.1. Basic Data in Two Groups. In the CKD group, there were 36 males and 24 females, aged $7.2 \pm 1.23$ years. The body mass index (BMI) was $20.31 \pm 3.3$. In the control group, there were 26 males and 19 females, aged $8.1 \pm 2.01$ years old, and the BMI was $21.28 \pm 2.94$. Their routine blood and urine tests, liver and kidney functions, electrocardiograms, and other tests were normal, and they had no history of diabetes, hypertension, heart failure, and other kidney diseases (Table 2). The CKD group was grouped according to eGFR, and the detection results showed that there were 7 cases of $\mathrm{CKD}$ stage I, 8 cases of CKD stage II, and 15 cases of CKD stage III-V each. According to the degree of kidney damage, there were 15 cases, 15 cases, and 30 cases, respectively (Table 3).

3.2. Analysis of SWE Ultrasound Image Results Based on DL. In Figure 3, the image effect processed by the DL-based SWE technology was greatly better than the traditional elastic ultrasound image, and the difference was statistically considerable $\left({ }^{*} P<0.05\right)$.

3.3. Comparison of YM Values of the Middle and Lower Kidneys. Comparison of YM values of the middle and lower kidneys in the CKD group and the control group showed that the elastic modulus values of the middle and lower parts of the left and right kidneys in the CKD group were 
TABLE 2: Basic data of children with CKD and control group.

\begin{tabular}{lcccc}
\hline & Male (cases) & Female (cases) & Age $($ cases $)$ & BMI $\left(\mathrm{kg} / \mathrm{m}^{2}\right)$ \\
\hline CKD group & 36 & 24 & $7.2 \pm 1.23$ & $20.31 \pm 3.3$ \\
Control group & 26 & 19 & $8.1 \pm 2.01$ & $21.28 \pm 2.94$ \\
\hline
\end{tabular}

TABLE 3: Pathological examination results of children in CKD group.

\begin{tabular}{ccccccccc}
\hline & \multicolumn{4}{c}{ Glomerular filtration rate } & \multicolumn{3}{c}{ Kidney damage degree } \\
& CKD1 & CKD2 & CKD3 & CKD4 & CKD5 & Mild & Medium & Severe \\
\hline Cases & 7 & 8 & 15 & 15 & 15 & 15 & 15 & 30 \\
\hline
\end{tabular}



FIgURE 3: Comparison of ultrasound imaging effects between the elastic ultrasound and algorithm-based $\operatorname{SWE}\left({ }^{*} P<0.05\right)$.

$(22.02 \pm 10.98) \mathrm{kPa}$ and $(21.99 \pm 11.87) \mathrm{kPa}$, respectively. Compared with the control group $(4.61 \pm 0.47) \mathrm{kPa}$ and $(4.50 \pm 0.59) \mathrm{kPa}$, those of the CKD group were substantially higher $(t=8.779$ and 10.362). From the comparison of the YM values of the middle and lower parts of the two kidneys, the difference was statistically considerable $(P<0.05)$ (Figure 4).

\subsection{Comparison of Young's Modulus in the Parenchyma of Both Kidneys in Different Stages of CKD}

3.4.1. Staging of Glomerular Filtration Rate. The YM of the middle and lower parenchyma of the left kidney was $5.32 \pm 1.05$ and $7.37 \pm 1.02$, and that of the right kidney was $6.37 \pm 1.15$ and $6.65 \pm 0.93$ in CKD stage $1-2$ patients, respectively. The difference was not statistically significant $(P>0.05)$. The YM of the middle and lower parenchyma of the left kidney in patients with CKD stages 3 to 5 were $13.27 \pm 0.83,24.21 \pm 5.69$, and $31.67 \pm 3.82$, respectively, and that of the right kidney was $17.26 \pm 0.98,26.76 \pm 7.22$, $32.37 \pm 4.27$, respectively, and the difference was significant $(P<0.05)$. With the increase of CKD stage, the degree of renal fibrosis between different groups was different, and the YM value of renal parenchyma gradually increased $(P<0.05)$, as explained in Figure 5.

3.4.2. Grading of Kidney Damage. The CKD group and the control group had remarkable differences in the YM values of the parenchyma of the middle and lower kidneys. The elastic shear wave values showed the right kidney $(F=329.45, \quad P<0.01)$ and the left kidney $(F=329.54$, $P<0.01)$. The YM of the middle and lower parenchyma of the CKD healthy group and the mild left kidney were $4.53 \pm 0.58$ and $5.63 \pm 1.18$, respectively, and that of the right kidney was $4.52 \pm 0.6$ and $5.75 \pm 1.22$, respectively. The difference was not statistically significant $(P>0.05)$. In patients with moderate and severe CKD, the YM of the middle and lower parenchyma of the left kidney was $17.27 \pm 0.83$ and $27.93 \pm 6.49$, respectively, and that of the right kidney was $17.26 \pm 0.98$ and $29.56 \pm 6.49$, respectively, with significant difference $(P<0.05)$. Accompanied by changes in the condition of the CKD group, the elasticity of the kidneys and the degree of fibrosis changed. The worsening of the disease caused the increase of kidney stiffness, and the YM value of kidney parenchyma also increased $(P<0.05)$ (Figure 6).

3.5. Differences in Serum Creatinine (Scr) and eGFR Levels in Each Phase of CKD Group. The Scr and eGFR levels of the control group were $66.44 \pm 7.34$ and $105.02 \pm 10.2$, respectively. The Scr levels of CKD 1-5 stages were 70.1 \pm 6.89 , $73.55 \pm 8.33, \quad 150.32 \pm 10.51, \quad 299.56 \pm 28.86, \quad$ and $531.78 \pm 48.65$, respectively; and the eGFR levels were $101.54 \pm 9.75,79.47 \pm 8.01, \quad 47.8 \pm 6.22,23.32 \pm 3.09$, and $6.76 \pm 1.83$, respectively. There was no statistically remarkable difference in eGFR in CKD stage I compared with the control group $(P>0.05)$. With the progress of CKD staging, the eGFR of CKD stages II-V was greatly lower than the control group, the difference was substantial $(P<0.05)$. In Figure 7 , there was no statistically remarkable difference between Scr in CKD stage I and the control group $(P>0.05)$. With the progress of CKD staging, the Scr in stages III-V increased greatly, and the difference was statistically considerable $(P<0.05)$ (Figure 8$)$. 


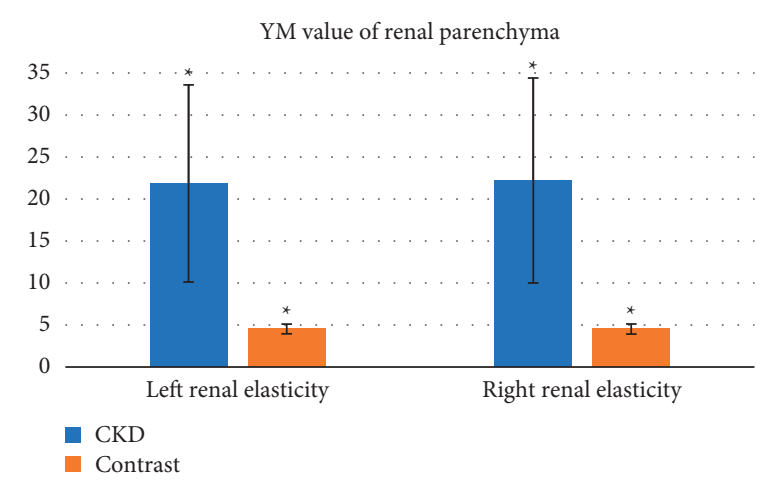

FIgURE 4: Comparison of YM in the middle and lower parts of the kidney between the control group and the CKD group $\left({ }^{*} P<0.05\right)$.

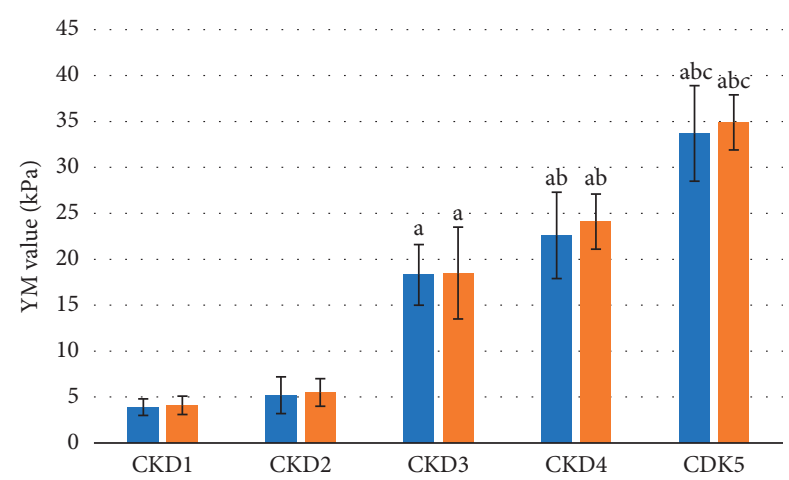

FIGURE 5: Comparison of renal parenchymal YM values in CKD stages 1-5. a: compared with CKD stage II, $P<0.05$; b: compared with CKD stage III, $P<0.05$; c: compared with CKD stage IV, $P<0.05$.



FIGURE 6: Comparison of YM values for different degrees of kidney damage. a: compared with healthy people, $P<0.01$; ab: compared with mild damage, $P<0.01$; abc: compared with moderate damage, $P<0.01$.

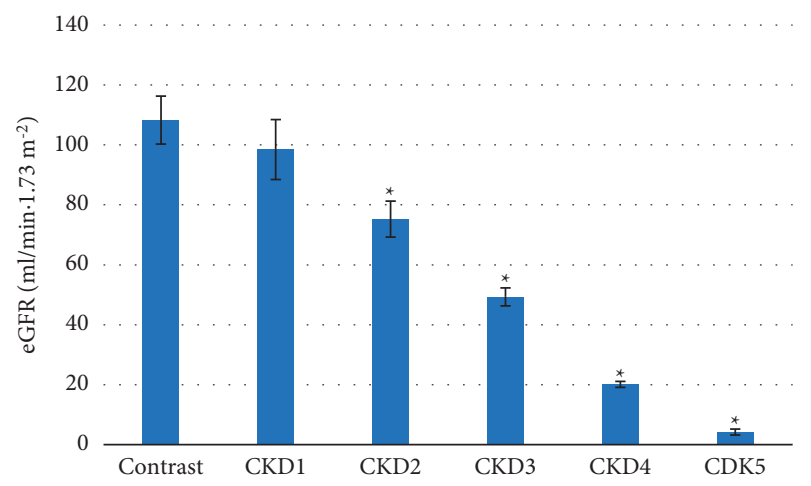

Figure 7: Comparison of eGFR levels in each phase of CKD and the control group. Compared with the control group, ${ }^{*} P<0.05$. 




FIGURE 8: Comparison of Scr levels in each stage of CKD and the control group. Compared with the control group, ${ }^{*} P<0.05$; compared with CKD stage II, $\# P<0.05$; compared with CKD stage III, $\$ P<0.05$; compared with CKD stage IV, $\& P<0.05$.

3.6. Analysis of Renal Parenchymal YM Values in Children with Membranous Nephropathy in Different Pathological Stages. Among the 60 children with CKD, 18 were pathologically diagnosed with membranous nephropathy, including 6 in stage I, 10 in stage II, and 2 in atypical membranous nephropathy. From the collected cases, no children had stage III or stage IV membranous nephropathy. After children with atypical membranous nephropathy were excluded, the renal parenchymal YM values of children with stage I and stage II membranous nephropathy were substantially higher than those of the control group, and there was a remarkable difference between the two groups $(P<0.05)$, as illustrated in Figures 9-11.

\section{Discussion}

The occurrence of $\mathrm{CKD}$ is closely related to glomerular function abnormalities, renal tubulointerstitial abnormalities, and renal vascular abnormalities. The main cause of death and disability in children is cardiovascular complications [18]. Primary CKD is pathologically irreversible. At present, the main treatment is finding out as early as possible and to intervene in time. In recent years, its incidence has become higher and the age of the patients is getting younger and younger, which seriously endangers human health, so it has become a disease of more concern in the medical field.

The SWE technology based on DL is a new and noninvasive diagnostic method. Compared with the traditional elasticity technology, it can quantitatively analyze the elasticity of the organization in real-time, without the need for an operator, and avoids the subjective and repeatable shortcomings of the scoring method $[19,20]$. The promotion of SWE technology also allows the tissue sclerosis information to be effectively measured, which has been proven to be very valuable for the diagnosis and evaluation of the severity of liver fibrosis [21]. It uses ultrasonic technology to evaluate and quantify the hardness or elasticity of the tissue, and accurate quantitative analysis results can be obtained. In addition, it has advantages over ultrasound examination results. Due to the relatively uniform propagation of ultrasound in different biological tissues, conventional

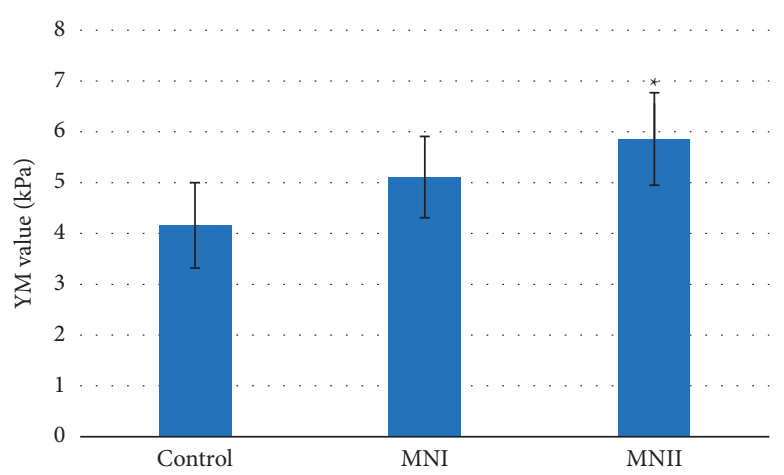

FIGURE 9: Comparison of renal parenchymal YM between children with membranous nephropathy and the control group $(* P<0.05)$.

ultrasound examinations cannot provide accurate information on the elastic characteristics of organs. However, because the anatomical characteristics of the kidney and the case problems involved in its insufficiency are very complicated, the SWE's research on this problem is still groundbreaking. The application of SWE technology based on DL in the diagnosis of organs and tissues is currently in its infancy, especially in terms of CKD staging and influencing factors. There are still uncertainties in the reasons for the impact of renal parenchymal elasticity. Although SWE technology has many shortcomings, it avoids the shortcomings of traditional CKD diagnosis and detection methods. It is expected to become a new noninvasive imaging technology for quantitative evaluation of CKD and an effective clinical examination method. In addition, it has been clinically applied to the diagnosis of breast, liver, thyroid, prostate, and other organs [22].

In this study, according to the comparison of the YM values of the middle and lower parenchymal kidneys of the CKD group and the control group, it was found that the length of the kidney and the parenchymal thickness of the lower pole of the children with CKD stages III-V in the CKD group were smaller than those of the control group. The kidney length and parenchymal thickness of the lower pole of children with CKD stage I and stage II were not greatly different from those of the control group. The results of staging nephropathy based on glomerular filtration rate showed that the SWE technique had good intergroup and intragroup reproducibility in measuring the kidneys of adults with CKD, but it was impossible to determine whether the kidney was in the early stage of CKD. It is generally believed that kidney damage is severe when GFR $<60 \mathrm{~mL} / \mathrm{min}$. Therefore, the YM values of the middle and lower kidneys of children with CKD stages III-V were compared, and the difference was considerable. It showed that real-time elastic shear wave elasticity can be used to detect middle- and latestage CKD.

This study has some limitations. This study takes children from a single hospital as the research object. The study period is short, the number of cases is small, and there may be a certain selection bias. The kidney is located at the back of the peritoneum and is deep in position, which is easily affected by respiration. The structure of the kidney is highly 


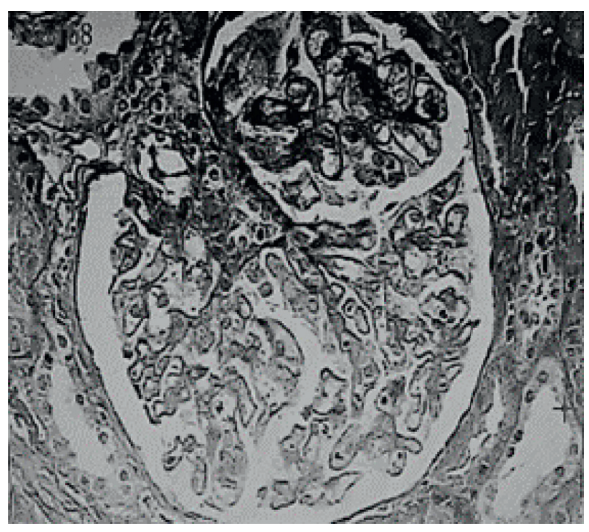

(a)

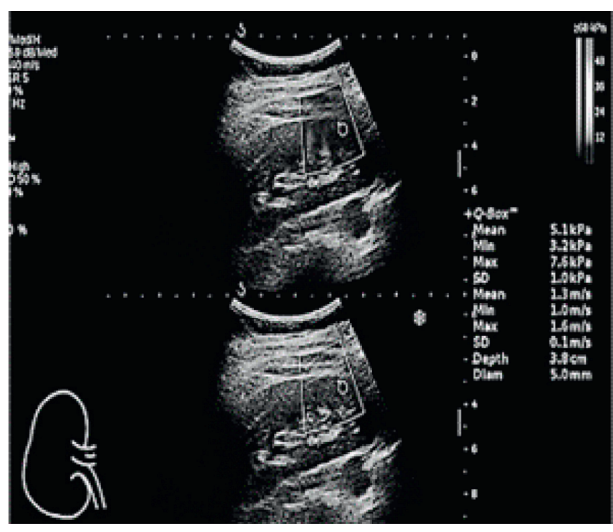

(b)

FIGURE 10: Stage I pathological picture of MN in children with CKD (a); YM of renal parenchyma (b).



(a)

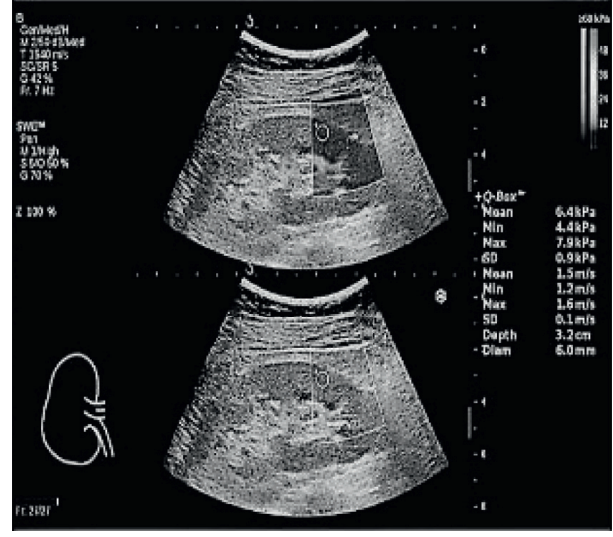

(b)

FIgURE 11: Pathological chart of stage II MN in children with CKD (a); YM of renal parenchyma (b).

anisotropic. The examination of the kidney by the SWE technology is affected by many factors, such as the child's breath-holding degree and physical fitness, the experience level of the operator, and the scanning technology. These factors will affect the accuracy of the measured value, but there is no unified standard operating method [23]. Therefore, a lot of clinical research and analysis is needed, which can provide a basis for the development and promotion of this technology and lay a solid theoretical technology.

\section{Conclusion}

The DL-based SWE technology can noninvasively and quantitatively distinguish the YM value and the degree of kidney damage in the kidney tissue of healthy people and CKD patients. As the stages of CKD increase, the degree of kidney damage gradually increases, the elasticity of the kidney tissue decreases, and the YM value increases. The YM value measured by DL-based SWE technology is related to biochemical indicators such as Scr and eGFR. The SWE technology based on DL is a new noninvasive, real-time, and quantitative detection method. It can effectively assess the stiffness of the kidneys, which can be used as a clinical reference to better detect the progress of CKD.

\section{Data Availability}

The data used to support the findings of this study are available from the corresponding author upon request.

\section{Conflicts of Interest}

The authors declare no conflicts of interest.

\section{Acknowledgments}

This work was supported by the Scientific research project of Hunan Provincial Health Commission (No. 20200434).

\section{References}

[1] D. A. Simonetto, P. Gines, and P. S. Kamath, "Hepatorenal syndrome: pathophysiology, diagnosis, and management," BMJ, vol. 370, 2020.

[2] J. Misurac, "Chronic kidney disease in the neonate: etiologies, management, and outcomes," Seminars in Fetal and Neonatal Medicine, vol. 22, no. 2, pp. 98-103, 2017. 
[3] V. A. Luyckx, D. Z. I. Cherney, and A. K. Bello, "Preventing CKD in developed countries," Kidney International Reports, vol. 5, no. 3, pp. 263-277, 2020.

[4] I. H. Lee, S. G. Kim, J. G. Kwon et al., "Intestinal tuberculosis in a patient with end-stage renal disease on hemodialysis," Medicine, vol. 99, no. 32, 2020.

[5] M. Kene, V. A. Arasu, A. K. Mahapatra, J. Huang, and M. E. Reed, "Acute kidney injury after CT in emergency patients with chronic kidney disease: a propensity scorematched analysis," Western Journal of Emergency Medicine, vol. 22, no. 3, pp. 614-622, 2021.

[6] R. F. Dubin, "Application of echocardiographic data in patients with chronic kidney disease," Current Opinion in Nephrology and Hypertension, vol. 27, no. 4, pp. 283-288, 2018.

[7] F. Abdolali, J. Kapur, J. L. Jaremko, M. Noga, A. R. Hareendranathan, and K. Punithakumar, "Automated thyroid nodule detection from ultrasound imaging using deep convolutional neural networks," Computers in Biology and Medicine, vol. 122, Article ID 103871, 2020.

[8] Z. Wang, L. Xie, and J. Qi, "Dynamic pixel-wise weightingbased fully convolutional neural networks for left ventricle segmentation in short-axis MRI," Magnetic Resonance Imaging, vol. 66, pp. 131-140, 2020.

[9] P. Kennedy, O. Bane, S. J. Hectors et al., "Magnetic resonance elastography vs. point shear wave ultrasound elastography for the assessment of renal allograft dysfunction," European Journal of Radiology, vol. 126, Article ID 108949, 2020.

[10] M. S. Garud and Y. A. Kulkarni, "Attenuation of renal damage in type I diabetic rats by umbelliferone - a coumarin derivative," Pharmacological Reports, vol. 69, no. 6, pp. 1263-1269, 2017.

[11] K. Vellanki and S. Hou, "Menopause in CKD," American Journal of Kidney Diseases, vol. 71, no. 5, pp. 710-719, 2018.

[12] V. V. B. Borisov and E. M. S. Shilov, "Chronic renal failure," Urologiia, vol. 1, no. 1, 18 pages, 2017.

[13] H. Jiang, Z. Feng, Z. Zhu et al., "Advances of the experimental models of idiopathic membranous nephropathy (Review)," Molecular Medicine Reports, vol. 21, no. 5, pp. 1993-2005, 2020.

[14] I. Petrucci, A. Clementi, C. Sessa, I. Torrisi, and M. Meola, "Ultrasound and color Doppler applications in chronic kidney disease," Journal of Nephrology, vol. 31, no. 6, pp. 863-879, 2018.

[15] X. Yang, F.-L. Hou, C. Zhao, C.-Y. Jiang, X.-M. Li, and N. Yu, "The role of real-time shear wave elastography in the diagnosis of idiopathic nephrotic syndrome and evaluation of the curative effect," Abdominal Radiology, vol. 45, no. 8, pp. 2508-2517, 2020.

[16] D. Abdelhafiz, J. Bi, R. Ammar, C. Yang, and S. Nabavi, "Convolutional neural network for automated mass segmentation in mammography," BMC Bioinformatics, vol. 21, no. 1, p. 192, 2020.

[17] H. El-Khatib, D. Popescu, and L. Ichim, "Deep learning-based methods for automatic diagnosis of skin lesions," Sensors, vol. 20, no. 6, p. 1753, 2020.

[18] M. Ruiz-Ortega, S. Rayego-Mateos, S. Lamas, A. Ortiz, and R. R. Rodrigues-Diez, "Targeting the progression of chronic kidney disease," Nature Reviews Nephrology, vol. 16, no. 5, pp. 269-288, 2020.

[19] S. X. Xie, Z. C. Yu, and Z. H. Lv, "Multi-disease prediction based on deep learning: a survey," Computer Modeling in Engineering and Sciences, vol. 127, no. 3, pp. 1-34, 2021.
[20] Y. Li, J. Zhao, Z. Lv, and J. Li, "Medical image fusion method by deep learning," International Journal of Cognitive Computing in Engineering, vol. 2, pp. 21-29, 2021.

[21] G. Ferraioli and R. G. Barr, "Ultrasound liver elastography beyond liver fibrosis assessment," World Journal of Gastroenterology, vol. 26, no. 24, pp. 3413-3420, 2020.

[22] Y. Sasaki, Y. Hirooka, H. Kawashima, T. Ishikawa, K. Takeshita, and H. Goto, "Measurements of renal shear wave velocities in chronic kidney disease patients," Acta Radiologica, vol. 59, no. 7, pp. 884-890, 2018.

[23] Y. Chen, B. Wang, L. Xiao, Y. Li, and Y. Luo, "Changes in muscle mass in patients with renal transplants based on ultrasound," Journal of Ultrasound in Medicine, vol. 40, no. 8, pp. 1637-1648, 2021. 